ILL-(TH) $-93-5$

DAMTP-93-16

\title{
The XY model on a dynamical random lattice
}

\author{
Simon M. Catterall \\ Department of Applied Maths and Theoretical Physics \\ University of Cambridge \\ Silver St. Cambridge CB3 9EW \\ England \\ John B. Kogut \\ Loomis Laboratory of Physics \\ University of Illinois at Urbana-Champaign \\ 1110 West Green Street \\ Urbana, Illinois 61801 \\ Ray L. Renken \\ Department of Physics \\ University of Central Florida \\ Orlando, Florida 32816
}

\begin{abstract}
We study the XY model on a lattice with fluctuating connectivity. The expectation is that at an appropriate critical point such a system corresponds to a compactified boson coupled to $2 \mathrm{~d}$ quantum gravity. Our simulations focus, in particular, on the important topological features of the system. The results lend strong support to the two phase structure predicted on the basis of analytical calculations. A careful finite size scaling analysis yields estimates for the scaling exponents in the low temperature phase.
\end{abstract}




\section{Introduction}

In recent years considerable progress has been made in understanding the nature of some simple systems encorporating two dimensional quantum gravity. These systems are thought to correspond to string theories out of the critical dimension. Two approaches have been followed which are at first sight quite different. The first of these employs techniques borrowed from conformal field theory to calculate the spectrum of anomalous dimensions in several simple models [1-2]. In the second method a regularisation of the continuum functional integrals is made in terms of random triangulations [3-5]. Where it has been possible to solve the models analytically the two approaches have been in complete agreement. Furthermore, the triangulated models may be studied nonperturbatively by numerical simulation. Whilst the analytical work gives only information on the scaling behaviour of matter field expectation values, the numerical studies allow us to probe the geometrical and fractal structure of the worldsheet. The lattice models may also be used for matter field central charges $c$ greater than unity where the continuum methods break down.

The marginal case when $c=1$ can be realised by employing a single scalar field. If the latter model is compactified by imposing that the field be periodic in some interval, no exact solution has been found. However there are strong theoretical arguments to suggest that the model may exist in two phases; one in which the scalar field is non-propagating and is described by pure $(c=0)$ gravity and another corresponding to a massless, propagating scalar field with unit central charge [6].

The lattice model is also interesting from a statistical mechanics point of view. The model in flat space (regular lattice) is known to undergo a Kosterlitz-Thouless KT phase transition at some critical coupling to a massless low temperature phase 
with a set of continuously varying critical exponents. This transition is driven by the condensation of topological defects (vortices) corresponding to field configurations with non-zero winding number. It is interesting to ask how this picture is modified by the encorporation of lattice (or metric) fluctuations. Naively, one might imagine that performing an annealed sum over lattices might strongly influence the effective forces between vortices and hence alter the character of any phase transition. Much of the results we present later will be concerned with elucidating the role such topological configurations play on a dynamical lattice.

\section{Discussion of the regular lattice model}

Consider initially the model on a fixed, regular lattice. The latter is taken to be the dual to a regular triangulation - a $\phi^{3}$-graph of toroidal topology and possessing $N$ nodes. The partition function defining the model is

$$
Z=\sum_{\theta_{i}} e^{-S(\beta)}
$$

with the lattice action $S(\beta)$ given by

$$
S(\beta)=-\beta \sum_{\langle i j\rangle} \cos \left(\theta_{i}-\theta_{j}\right) \quad \theta_{i} \in 0 \cdots 2 \pi
$$

Using techniques drawn from conformal field theory it is possible to show that the critical region of this theory can be described in terms of two sets of operators with anomalous dimensions $\left\{\Delta_{n}, \bar{\Delta}_{m}\right\}$ (see for example [7]).

$$
\begin{aligned}
& \Delta_{n}=\frac{1}{2}\left(\frac{1}{2 \pi \beta_{\text {eff }}}\right) n^{2} \\
& \bar{\Delta}_{m}=\frac{1}{2}\left(2 \pi \beta_{\text {eff }}\right) m^{2}
\end{aligned}
$$

The former operators $\left(\Delta_{n}\right)$ correspond to spin waves, whilst the latter $\left(\bar{\Delta}_{m}\right)$ are to be identified with vortices. The effective coupling constant $\beta_{\text {eff }}$ is determined 
by details on the scale of the lattice cutoff, for example the lattice type and bare lattice coupling $\beta$. However, for large coupling $\beta$ we expect

$$
\beta_{\mathrm{eff}} \sim \beta, \quad \beta \rightarrow \infty
$$

The vortex operators are seen to be irrelevant operators at large $\beta_{\text {eff }} \sim \beta$ and the critical theory can be understood in terms of its spin wave content. In this region we expect that the theory is massless and the two point function corresponding to spin wave excitations behaves as

$$
\left\langle e^{i \theta(0)} e^{-i \theta(r)}\right\rangle \sim r^{-2 \Delta_{1}} \sim r^{-\frac{1}{2 \pi \beta_{\mathrm{eff}}}}
$$

However for small $\beta$ this situation is reversed and the vortex operators are relevant and dominate the long distance physics. A phase transition is thus expected between a region where spin waves essentially exhaust the physically relevant degrees of freedom and one where the ground state is populated by vortices. This transition will occur when the the anomalous dimension of the lowest vortex operator $\bar{\Delta}_{1}$ is just marginal $\left(\bar{\Delta}_{1}=2\right)$. This yields an estimate for the critical coupling

$$
\beta_{\mathrm{eff}}^{c}=\frac{2}{\pi}
$$

A careful renormalisation group analysis [8] shows that the phase transition at $\beta_{c}$ is of an unusual type - the free energy and all its derivatives are continuous at $\beta_{c}$ whilst the correlation length and susceptibility possess essential singularities as the transition is approached from the disordered phase.

$$
\begin{aligned}
& \xi \sim a e^{\frac{b}{\left(\beta_{c}-\beta\right)^{\nu}}} \\
& \chi \sim c e^{\frac{d}{\left(\beta_{c}-\beta\right)^{\nu}}}
\end{aligned}
$$

The exponent $\nu$ takes the value $\nu=1 / 2$. Furthermore, the dimension of the lowest spinwave operator is then

$$
\Delta_{1}=\frac{1}{8}, \quad \beta=\beta_{c}
$$


The presence of such a phase transition can be inferred in another way by considering the free energy of such a vortex configuration. At large $\beta$ the cosine action may be replaced by an equivalent gaussian action, provided we retain the periodicity of the $\theta$ coordinates. A simple vortex configuration would be $\theta=m \phi$ in an $(r, \phi)$ polar coordinate system. This yields an action for the vortex of

$$
S_{\mathrm{vortex}}=-\beta_{\mathrm{eff}} \pi n^{2} \ln \left(\frac{L}{a}\right)
$$

where $L$ is the infrared and $a$ the ultraviolet cutoff. Since there is a choice of $(L / a)^{2}$ positions on a cubic lattice at which to place the centre of such a vortex (with $n=1$ ) the contribution to the free energy of such a configuration is

$$
\delta F=\left(\pi \beta_{\mathrm{eff}}-2\right) \ln \left(\frac{L}{a}\right)
$$

Clearly for large $\beta_{\text {eff }}$ vortices will be irrelevant, whilst for small coupling they will dominate, disordering the ground state and forcing a finite correlation length.

The presence of such non-trivial field configurations is signaled by a non-zero vorticity $V$ defined in the continuum by

$$
V=\frac{1}{2 \pi} \int_{C} \nabla \theta \cdot d s
$$

We have employed the naive transcription of this object onto the lattice

$$
V=\sum_{\text {loops }}^{N_{L}} \sum_{j}\left(\theta_{j+1}-\theta_{j}\right) \bmod 2 \pi
$$

The outer sum is carried out over all the $N_{L}$ loops of the $\phi^{3}$-graph, whilst the inner sum corresponds to computing the net change in angle on traversing a given loop. In practice, the total vorticity vanishes as a consequence of the boundary conditions, so we measure the modulus of the local vorticity in our simulations. Clearly, at large $\beta$, where all the spins are locally correlated, this is likely to be 
a good approximation to the continuum expression, but one might worry about the situation at small coupling. This is, of course, the usual problem of defining topology on a lattice. The problem is to construct lattice observables which go smoothly over into continuum topological quantities. Typically, the lattice operators receive large contributions from fluctuations at order the cutoff which have no place in the continuum expectation values. One of the standard methods to measure topological charge for lattice QCD is by a so-called cooling method [9], whereby the action is locally minimised to remove ultraviolet fluctuations. We have tested such a method in this situation and found that for a wide range of couplings close to criticality the winding number remains constant under cooling giving us confidence that we are not seeing lattice artefacts.

\section{Fluctuating lattice model}

The simulations described later will concern themselves with a nonperturbative study of this model when formulated on a dynamical random lattice. As we have described this prescription is equivalent (at least when $c<1$ ) to coupling the spin model to gravity. Since the XY model has $c=1$ at large $\beta$, the KPZ framework [1] marginally applies and we can attempt to derive some predictions for this model in the presence of such metric fluctuations. Specifically, if we take some operator $O(x)$ and integrate it over the surface (to get a reparametrisation invariant object), the expectation value will scale with surface area as

$$
\left\langle\int d^{2} x \sqrt{g} O(x)\right\rangle_{\text {gravity }} \sim A^{1-D}, \quad A \rightarrow \infty
$$

The KPZ formula then relates this gravitational scaling dimension $D$ to the anomalous dimension $\Delta$ of $O(x)$.

$$
D=\sqrt{\Delta / 2}, \quad c=1
$$


Similarly, an integrated correlation function or susceptibility conjugate to $O(x)$ would scale like

$$
\left\langle\int d^{2} x \sqrt{g} O(x) \int d^{2} x^{\prime} \sqrt{g^{\prime}} O\left(x^{\prime}\right)\right\rangle_{\text {gravity }} \sim A^{(2-2 D)}
$$

To adopt a conventional normalisation of the susceptibility we would divide by a power of the area to get

$$
\chi(O) \sim A^{(1-2 D)}=A^{(1-\sqrt{2 \Delta})}
$$

which is to be compared with the usual finite size scaling in the absence of gravitational fluctuations

$$
\chi^{\text {flat }}(O) \sim A^{(1-\Delta)}
$$

For the gravity coupled system the condition for relevance of an operator $O$ is now that its gravitational scaling dimension $D(O)=1$, which in the case of the vortex operators $O=\bar{\Delta}_{1}$ yields the condition that $\bar{\Delta}_{1}=2$. This is precisely the same condition that determined the critical coupling in flat space. Thus this continuum analysis would predict that the gravitational dressing of the vortex operators does not change the position of the phase transition. However it is not clear that the transition is still of KT type. We will address this question again when interpreting our results.

The simulation of this dynamical model is effected by means of two forms of update. To simulate the sum over spin configurations we have employed the cluster update pioneered by Wolff [10]. This proves to be a very efficient method for quasimassless theories like the XY model. The sum over graphs is effected by a local procedure detailed in a previous paper [11]. We have simulated lattice volumes from $N=100$ to $N=5000$, typically using $O\left(10^{5}\right)$ sweeps of the lattice per coupling $\beta$. Errors were assessed by the usual rebinning procedure. 


\section{Results and analysis}

Since the continuum arguments suggest that the transition on the dynamical lattice may be rather similar to the regular (flat space) theory, we chose to do some moderate simulations of the latter to compare with the dynamical results. Fig.1 shows a plot of the specific heat $C$

$$
C=\frac{1}{N}\left(\left\langle S^{2}\right\rangle-\langle S\rangle^{2}\right)
$$

as a function of lattice coupling $\beta$. The plot also shows the topological susceptibility $\chi_{v}$ determined by

$$
\chi_{v}=\frac{1}{N_{L}}\left(\left\langle V^{2}\right\rangle-\langle V\rangle^{2}\right)
$$

Clearly, both show peaks in the neighbourhood of $\beta_{c} \sim 1.6$ which may be taken as a signal for some form of critical behaviour. However, the specific heat rapidly saturates with increasing system volume and hence is not a good observable for determining the nature of any phase transition. Furthermore, in the case of the dynamical system the peak in $C$ occurs at a substantially different coupling than our best estimate of $\beta_{c}$. Similarly, the spin susceptibility increases monotonically with $\beta$ and yields no information on the position of any phase transition.

In contrast, the topological susceptibility being determined by the degrees of freedom which are believed to drive the phase transition, is a much more reliable indicator of criticality in the system. Indeed, we have taken the peak in $\chi_{v}$ as our best estimate of the critical point. From the largest regular lattices we have considered we estimate the critical lattice coupling to be $\beta_{c}=1.7(1)$. As we detail below this produces estimates for the critical exponents on a regular lattice which are in agreement with analytical calculations.

Fig 2. shows the vorticity $V$ for a 3000 node dynamical lattice over a range of 
couplings spanning the critical region. The absence of any discontinuities makes it unlikely that there are any first order transitions in the system. However the topological susceptibility is much more interesting and is shown in fig. 3 for the full range of system sizes we studied. The first point to note is that the critical coupling, as dictated by the peak in $\chi_{v}$, has substantially shifted from its value on the regular (flat space) lattice $\beta_{c}=1.7$ to a value which we estimate from the peak on the largest lattice to be $\beta_{c}=2.3(1)$. Note that this is in agreement with an earlier study which utilised an entirely different method to derive a value for $\beta_{c}$ $[12]$

However, at first sight, this renormalisation of the coupling would appear to be at variance with the continuum calculations which indicate that transition coupling is not changed by the metric fluctuations. In an effort to cast some light on this we simulated an XY model on a single quenched lattice deriving from a simulation of pure gravity. In this case the peak in $\chi_{v}$ again occurred in the vicinity of $\beta_{c}=2.3$. We interpret this situation in the following way. The transition occurs at some value of $\beta_{\text {eff }}$ which is identical on both flat and fluctuating geometries. However the corresponding bare lattice coupling may be different in the regular and dynamical lattice cases (since their local lattice structure is radically different). Furthermore, it is then conceivable that the KPZ predictions (eqn. 18) for the fluctuating lattice will still hold true at the appropriate critical point (and indeed our later results will lend support to this conclusion).

The second observation to be made from fig. 3 is that $\chi_{v}$ shows strong finite volume behaviour for large $\beta$. This is also true of the model restricted to a regular lattice and comes about for the following reason. Consider the regular lattice topological susceptibility $\chi_{v}^{\text {flat }}$ as a (euclidean) time $t$ integral over a vortex-vortex 
correlator.

$$
\chi_{v}^{\mathrm{flat}}=\sum_{t} \sum_{s}\left|\left\langle v_{0} \mid s\right\rangle\right|^{2} e^{-E_{s} t}
$$

The states $s$ are eigenstates of the lattice Hamiltonian with energies $E_{s}$. Clearly the integral will be dominated by the lightest state $s_{0}$ which couples to the local vortex operator $v_{0}$. It will be this state which will govern the finite size scaling of the susceptibility. If we regard the matrix elements $\left\langle v_{0} \mid s\right\rangle$ now as expectation values in a path integral, we can use general symmetry principles to determine which of them, in principle, are nonvanishing. Consider the overlap of a spinwave state $s=e^{i \theta}$ on the local vorticity $v_{0}$. If we perform a parity transformation of the form

$$
x_{1} \rightarrow-x_{1}, \quad x_{2} \rightarrow x_{2}, \quad \theta \rightarrow \theta
$$

the vorticity changes sign but the spin wave state is invariant. Thus, provided parity is not spontaneously broken, the spinwave and vortex sectors are completely orthogonal. However if we take as operator the modulus of the local vorticity (which is the case in our numerical simulations) then both operators are invariant under this parity transformation and hence we would expect on these general grounds that the lightest state coupling to such an operator would again be the simple spin wave state. Thus the finite size scaling of the topological susceptibility will be governed by the same critical exponent $\Delta_{1}$ as for the spin susceptibility. Similarly in the gravitationally dressed case of the dynamical ensemble the two susceptibilities will scale with the same exponent. A measurement of both quantities gives us independent estimates of this scaling exponent.

This picture is borne out by our numerical data. Fig. 4 reveals an analysis of the finite volume behaviour of the spin susceptibility $\chi_{s}$ for both regular and fluctuating lattices. As we emphasised, we expect that its finite size scaling will be dominated by the lightest spin wave state $(n=1)$. 
The regular lattice data shown derive from runs at the transition point $\beta_{c}=1.7$ We fit our data from lattice sizes $N=968$ to $N=5000$ to the form

$$
\chi \sim a+b N^{\omega}
$$

using a simple nonlinear least squares procedure. The fit yields $\omega^{\text {flat }}=0.855(3)$ with $\chi^{2} /$ dof $=0.4$ with $55 \%$ confidence. This number is to be contrasted with the prediction $\omega^{\text {flat }}=0.875$ dictated by the usual KT and CFT arguments. However the fluctuating lattice gives the fit $\omega=0.53(13)$ with $\chi^{2} /$ dof $=0.04$ at $85 \%$ confidence. This, in turn, lies remarkably close to the KPZ prediction of $\omega=0.5$ derived from eqn. 18 with $\Delta_{1}=1 / 8$.

Similar conclusions can be drawn in the case of the topological susceptibility $\chi_{v}$ (fig. 5). Here, the regular lattice fit gives $\omega^{\text {flat }}=0.843(31), \chi^{2} /$ dof $=0.02$ with a confidence level of $89 \%$, which is consistent with the spin susceptibility measurement and compatible with the theoretical prediction. The dynamical lattice yields $\omega=0.64(15), \chi^{2} /$ dof $=0.1$ at $79 \%$ confidence. This again is in reasonable agreement with the KPZ number of 0.5 and is inconsistent (at two standard deviations) with the flat space scaling exponent $\omega=0.875$.

Clearly then, we have seen very good agreement with the usual KT assignments in the case of flat space. This gives us confidence that our method for locating the transition coupling is a good one. Furthermore, the dynamical exponents are dramatically different and lie intriguingly close to the naive KPZ prediction of the continuum theory, even the critical lattice coupling for the dynamical ensemble is significantly different from its flat space value.

As an independent check we have examined the spin correlator $g(r)$ for the regular lattice at our best estimate for $\beta_{c}=1.7$. Fig.6 shows an 'effective' $\eta$-plot determined from the spin correlator on a lattice with $N=2888$ by assuming power 
law behaviour

$$
\eta(r)=\frac{\ln \left(\frac{g(r+1)}{g(r)}\right)}{\ln \left(\frac{r+1}{r}\right)}
$$

We expect finite size effects to be important when $r>15$ and lattice spacing errors to be present for small $r$, but the figure clearly shows a rather stable plateau for $\eta$ within this region. Indeed the plot of fig. 7 allows a fit for $\eta$ in this range yielding $\eta=0.247(1)$ with $\chi^{2} /$ dof $=1.4$. This is to be compared with the predition of $\eta=2 \Delta_{1}=0.25$ from KT theory. The 'effective' mass computed in the analogous way on the assumption of an exponential behaviour for $g(r)$ is shown also in fig. 6. Clearly there is no stable region for fitting here and we interpret this as strong indication that at the critical point of the regular lattice XY model there are power law correlations.

Contrast these conclusions with fig. 8 which shows the same quantities computed with the ensemble of random graphs. In this case we have to be more careful in defining the correlation function $g(r)$. On the lattice we define $g$ as follows

$$
g(r)=\left\langle\frac{1}{n(r)} \sum_{i j} \sigma_{i} \sigma_{j} \delta\left(d_{i j}-r\right)\right\rangle
$$

The function $d_{i j}$ is just the geodesic distance (minimal length walk) on a given graph between sites $i$ and $j$, whilst

$$
n(r)=\sum_{i j} \delta\left(d_{i j}-r\right)
$$

We average over spin configurations $\sigma_{i}=e^{i \theta_{i}}$ and graphs $G_{N}$. For a fixed lattice this just reduces to the usual definition of the propagator. Notice that the continuum version of this quantity is just

$$
g(r)=\left\langle\frac{1}{n(r)} \int d^{2} x \int d^{2} x^{\prime} \sqrt{g} \sqrt{g^{\prime}} \phi(x) \phi\left(x^{\prime}\right) \delta\left(d\left(g, x, x^{\prime}\right)-r\right)\right\rangle_{\phi+\operatorname{grav}}
$$


This correlation function is explicitly reparametrisation invariant (if the fields $\phi$ are scalars) since the identity and length of the geodesics $d\left(x, x^{\prime}, g\right)$ are reparametrisation invariant. Therefore this correlation function is a bona fide observable for gravity coupled systems. However, in this dynamical case, the effective power fit plot at $\beta=2.3$ (fig. 8), is extremely poor, and the exponential fit proves considerably better. Indeed, the best fit we could produce (fig. 7) corresponded to a two exponential fit from distances 3 to 15 of the form

$$
g(r)=a \exp -b r+c \exp -d r
$$

with the results $a=0.23(1), b=0.32(4), c=0.69(2)$ and $d=0.048(2)$ with a $\chi^{2} /$ dof $=0.28$ at $97 \%$ confidence. The second exponential presumably corresponds to an excited state, but at present it is not at all clear why there is this qualitative difference in the structure of this correlation function $g$ when the system is coupled to gravity.

We have also attempted to fit the spin susceptibility for the dynamical lattice simulations to the KT form of essential singularity. Using the data at $N=3000$ and with $\beta_{c}=2.3$ as before, we have assumed $\nu=0.5$ and attempted to fit the data in the disordered phase. Fig. 9 illustrates this by plotting $\ln \chi_{s}$ as a function of $\left(\beta_{c}-\beta\right)^{-0.5}$. The rounding visible in the plot corresponds to the onset of finite size effects, but clearly the data set is not inconsistent with a KT type singularity. However, it is perfectly possible to fit the data reasonably well over the same range by a simple power fit and objectively it is very difficult to assess which is the better. This situation is well known in the case of the ordinary XY model and it is only relatively recently that numerical simulations have proven capable of resolving this problem [13]. With the lattice sizes employed in this study, such a definitive 
conclusion is impossible. However we can say that a fit of the form

$$
\chi \sim a\left(\beta_{c}-\beta\right)^{-\gamma}
$$

requires, typically, a very large coefficient $a \sim 40.0$ and power $\gamma=3.5-4.0$.

To examine the effective gravitational action induced by the coupling of spins to the dynamical lattices, we also measured the fluctuation in the local scalar curvature. This is just defined by

$$
r^{2}(\beta)=\left\langle\sum_{i}^{N_{L}} \frac{\left(6-l_{i}\right)^{2}}{l_{i}}\right\rangle
$$

where $l_{i}$ is the length of loop $i$ and the sum runs over all loops in the $\phi^{3}$-graph. Fig 10. shows this as a function of the bare lattice coupling for a variety of lattice volumes. Clearly finite size effects are small, and the coupling to matter enhances the production of singular geometries. Notice that the the effects of this are almost constant throughout the low temperature phase, perhaps suggesting that, as has been observed before [14,15], the effective action for gravity may be governed only by the central charge; the field content and anomalous dimensions seem to have little influence on the gravitational sector (at least in those models examined so far). The figure also shows the same quantity for two Ising models confirming this universality.

On the same figure we show a quantity $W$ corresponding to the cross correlator of the local vorticity (again its modulus strictly) with the ring length.

$$
W(\beta)=\frac{\left\langle\sum_{i}^{N_{L}} l_{i}\left|v_{i}\right|\right\rangle}{\sum_{i}^{N_{L}}\left\langle l_{i}\right\rangle\left\langle\left|v_{i}\right|\right\rangle}-1
$$

The signal is normalised by the disconnected part to factor off the trivial $\beta$ dependence of the vorticity. Again a peak is seen close to the initial peak in the scalar curvature fluctuation $r^{2}$ and significantly to the left of the estimated 
transition point. This would indicate a significant tendency of vortices to bind to curvature defects on the lattice. The latter can be understood qualitatively as a consequence of the logarithmic divergence of the free energy of a single vortex. The free energy is lowered if the vortex forms on a ring of large length (the ultraviolet cutoff a in eqn. 11). This conclusion appears to be essentially unaltered when fluctuations are taken into account.

Finally we have studied the clustering properties of vortices and antivortices. To do this we introduce the joint probability distributions $P_{v v}(r)$ and $P_{v a v}(r)$. If we have a vortex at the origin $r=0$, these measure the probabilities of finding a vortex (in the case of $\left.P_{v v}\right)$ or antivortex $\left(P_{v a v}\right)$ at some geodesic distance $r$ (measured with the respect to the loops). Fig. 11 illustrates this with histograms of these distributions in the disordered phase and ordered phase. Clearly the vortex-vortex distribution is essentially the same in both cases and corresponds to an approximately uniform distribution of vortices over the plane. However a dramatic difference is seen in the case of $P_{v a v}$. In the disordered phase the there is a no evidence of any real clustering, whilst in the low temperature phase almost all antivortices are bound one lattice spacing away. This is completely analogous to the situation in flat space where the disordered phase corresponds to a uniform plasma of vortices and antivortices, whilst in the low temperature phase the vortices and antivortices exist only in bound pairs. The transition is then pictured in this language as the dissociation of these dipole-like pairs, whilst the peak in $\chi_{v}$ corresponds to a maximal fluctuation between pairs as their effective binding decreases to zero. Thus, the qualitative picture of a transition driven by condensation of defects persists even when the defects are dressed by gravitational fluctuations. 


\section{Conclusions}

We have presented results for the XY model coupled to a fluctuating lattice of fixed topology. These simulations are motivated by the need to understand the coupling of $2 \mathrm{~d}$ gravity to simple matter systems. We have collected data both for the standard spin wave observables and also the topological sector. The estimates of the critical exponents we derive from finite size scaling analyses are in agreement with both flat space KT predictions and the KPZ framework.

We observe that the fluctuations in the geometry plateau in the low temperature phase at a value commensurate with a two Ising system which is consistent with the assignment of central charge one for this phase. Furthermore we observe a strong binding of the vortices to regions of high curvature close to the critical point. The distribution functions for vortex-vortex and vortex-antivortex pairs confirm the qualitative picture of the two phases as given by KT theory in flat

space. The presence of metric fluctuations, whilst dressing operators, seems to preserve the nature of the transition.

These results then confirm the phase structure argued for on the basis of continuum calculations, and furnish yet further evidence of the validity of the dynamical triangulation prescription (and its computer implementation) in the marginal case of $c=1$ theories.

This work was supported, in part, by NSF grant PHY 92-00148 and some of the numerical calculations were performed using the Florida State University CRAY YMP. We acknowledge fruitful discussions with Ian Drummond and Sumit Das. 


\section{REFERENCES}

[1] V. Knizhnik, A. Polyakov and A. Zamolodchikov, Mod. Phys. Lett.A3 8191988.

[2] J. Distler and H. Kawai, Nucl. Phys. B321, 509 (1989).

[3] J. Ambjorn, B. Durhuus and J. Frohlich, Nucl. Phys. B257, 433 (1985).

[4] F. David, Nucl. Phys. B257, 543 (1985).

[5] B. Boulatov, V. Kazakov, I. Kostov and A. Migdal, Nucl. Phys. B275, 641 (1986).

[6] D. Gross and I. Klebanov, Nucl. Phys. B344, 475 (1990).

D. Gross and I. Klebanov, Nucl. Phys. B354, 475 (1990).

D. Boulatov and V. Kazakov, Nucl. Phys. B (Proc. Suppl.) 25A (1992) 38.

[7] Itzykson and Zuber, Statistical Field Theory II, CUP 1989.

[8] J. Kosterlitz and D. Thouless, J. Phys. C6 (1973) 1181.

J. Kosterlitz, J. Phys. C7, (1974), 1046.

[9] M. Teper, Phys. Lett. B202 (1988) 553.

[10] U. Wolff, Phys. Rev. Lett. 62 (1989) 361.

[11] S. Catterall, D. Eisenstein, J. Kogut and R. Renken, Nucl. Phys. B366, 647 (1991).

[12] C. Baillie and D. Johnston, COLO-HEP-286.

[13] U. Wolff, Nucl. Phys. B322, 759 (1989).

R. Gupta and C. Baillie, Phys. Rev B45 (1992) 2883. 
[14] C. Baillie and D. Johnston, COLO-HEP-276.

G. Thorleifsson, Nucl. Phys. B (Proc. Suppl.) 30 (1993) 787.

[15] S. Catterall, R. Renken and J. Kogut, Phys. Lett. B292, (1992) 277. 


\section{FIGURE CAPTIONS}

[1] Specific Heat $C$ and Topological Susceptibility $\chi_{v}$ for a regular lattice $(N=968)$ as a function of bare coupling $\beta$.

[2] Vorticity on a 3000 node dynamical lattice vs $\beta$.

[3] Topological Susceptibility $\chi_{v}$ on a dynamical lattice. Lattice volumes are $N=100$ $(\times), N=1000(\triangle) N=2000(\diamond), N=3000(\square), N=5000($ ( ).

[4] Spin Susceptibility $\chi_{s}$ for both regular (०) and fluctuating ( $\left.\diamond\right)$ lattices vs number of nodes $N$.

[5] Topological Susceptibility $\chi_{v}$ for both regular (॰) and fluctuating ( $\left.\diamond\right)$ lattice vs number of nodes $N$.

[6] Power (o) vs exponential ( $\square$ ) fits for a regular lattice $N=2888$ at $\beta=1.7$.

[7] Spin Correlators for regular and fluctuating lattices at criticality together with power law and exponential fits respectively.

[8] Power (o) vs exponential ( $\square$ ) fits for a dynamical lattice $N=3000$ at $\beta=2.3$.

[9] $\log \left(\chi_{s}\right)$ vs $t^{-1 / 2}, t=\beta_{c}-\beta$, together with KT linear fit.

[10] Fluctuation in local scalar curvature $r^{2}$ and cross correlator of vorticity with loop length $W$ vs $\beta$.

[11] Histograms of joint probability distributions for vortex-vortex $\left(P_{v v}(r)\right)$ and vortexantivortex $\left(P_{\text {vav }}(r)\right)$ pairing for $\beta=3.0$ and $\beta=1.0$. 


\section{Topological Susceptibility and Specific Heat}

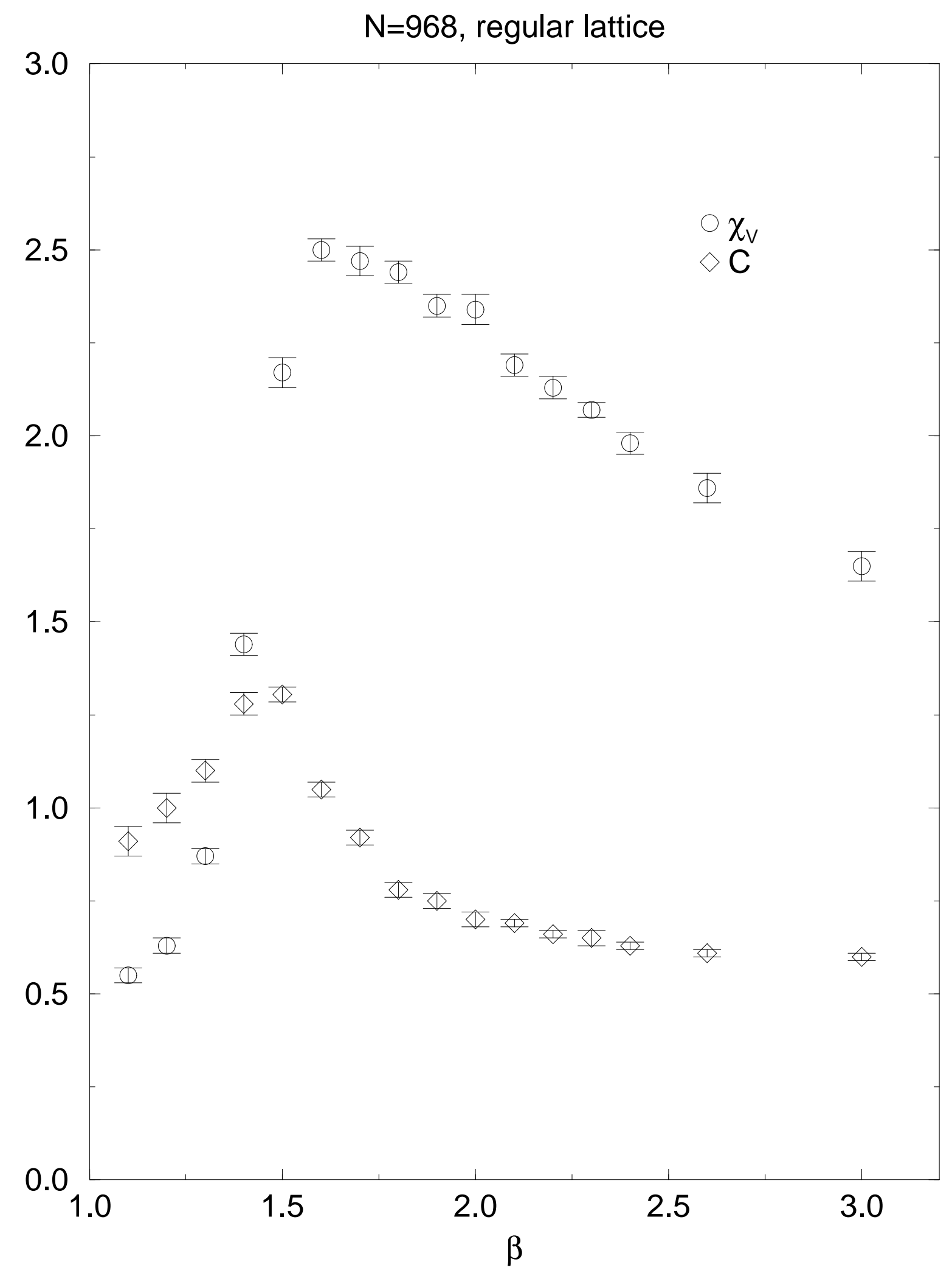




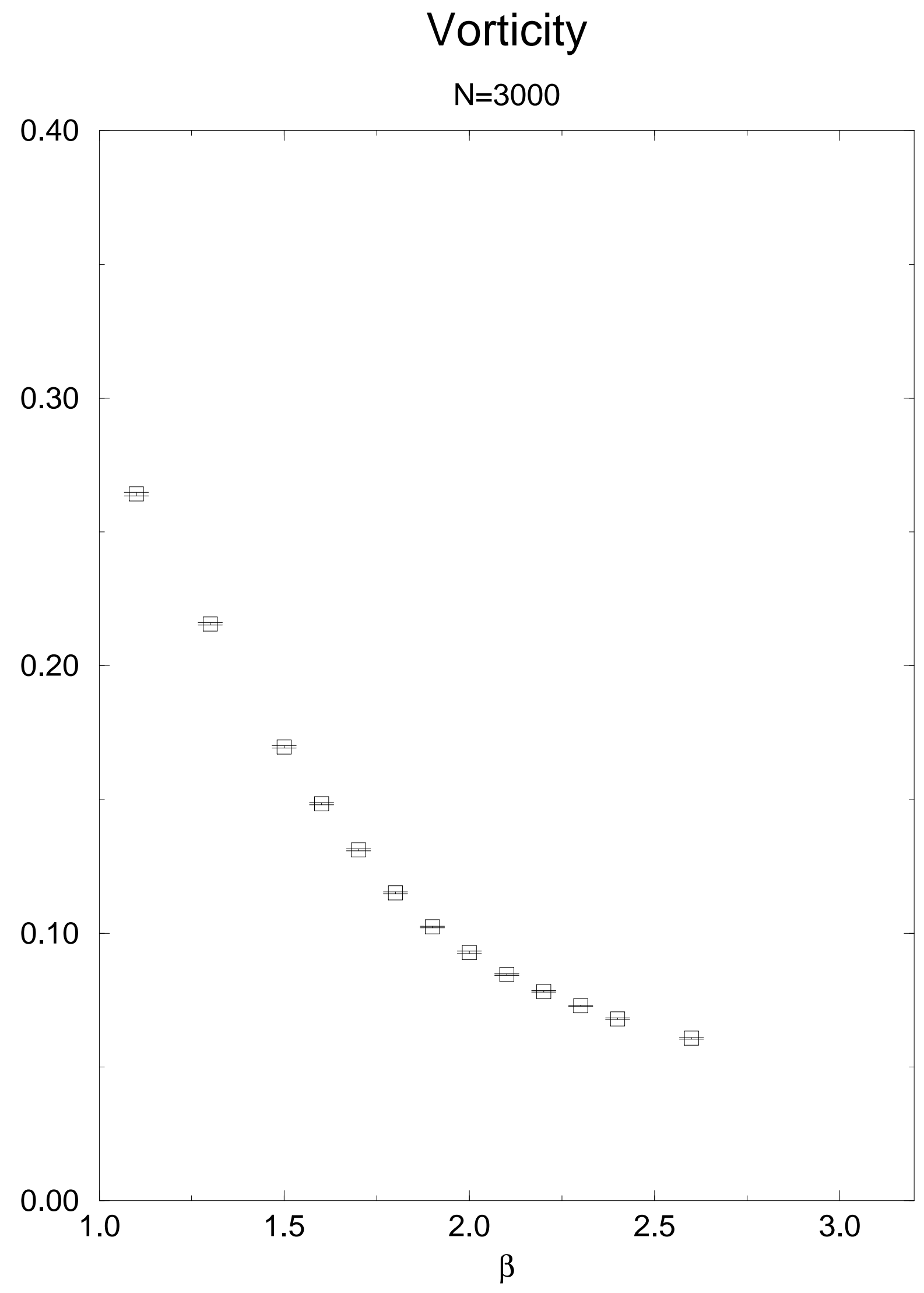




\section{Topological Susceptibility}

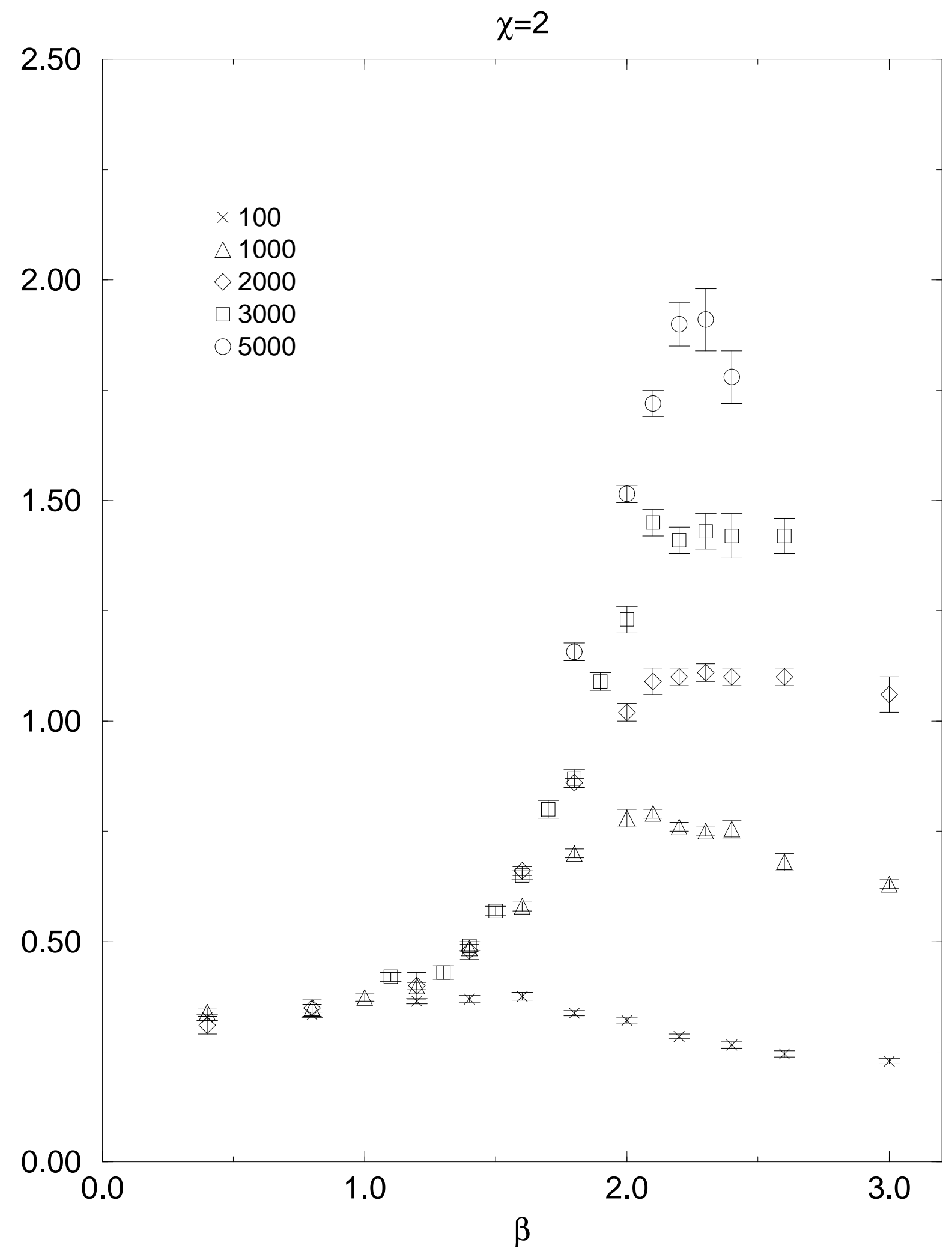




\section{Spin Susceptibility}

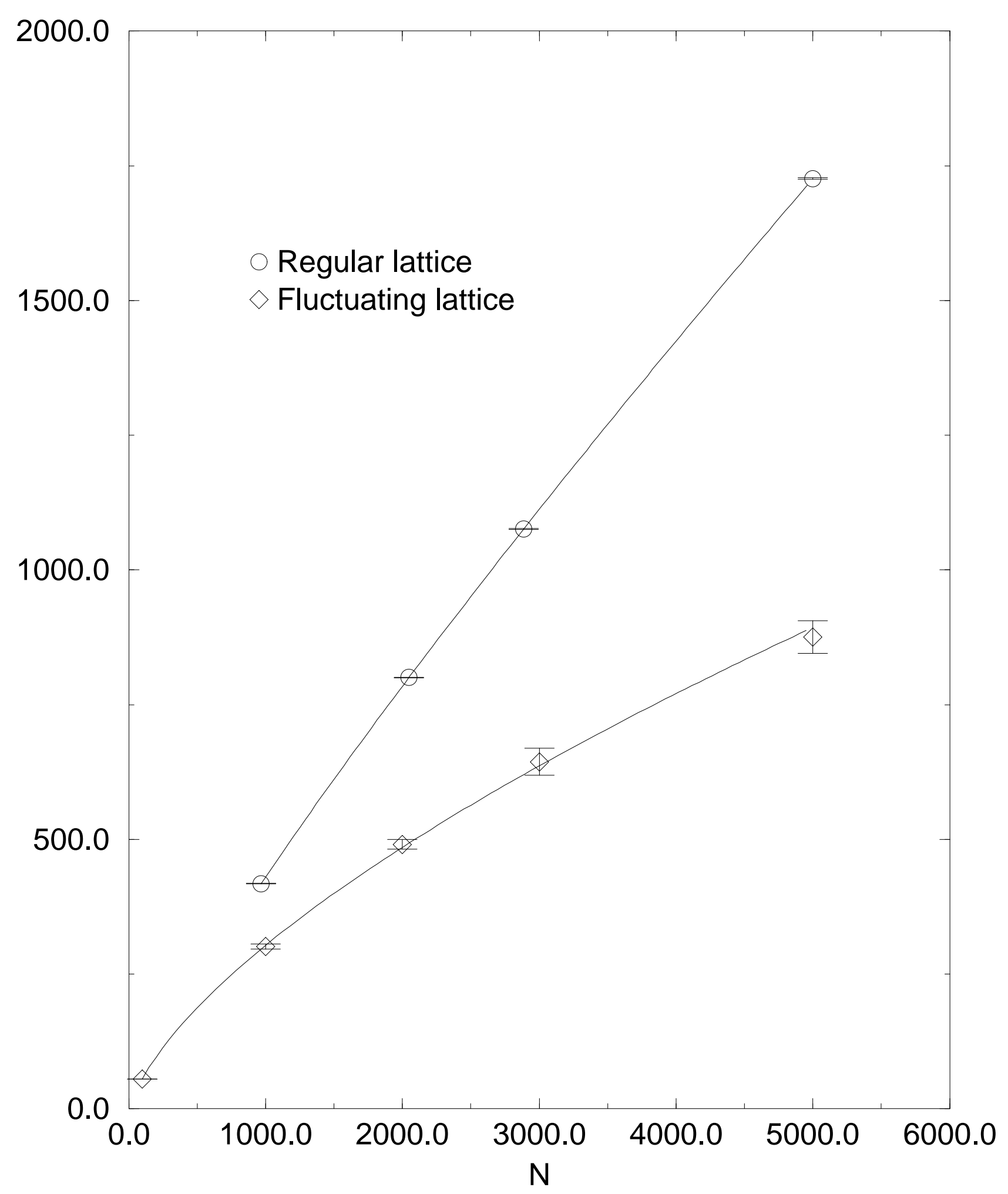




\section{Topological Susceptibility}

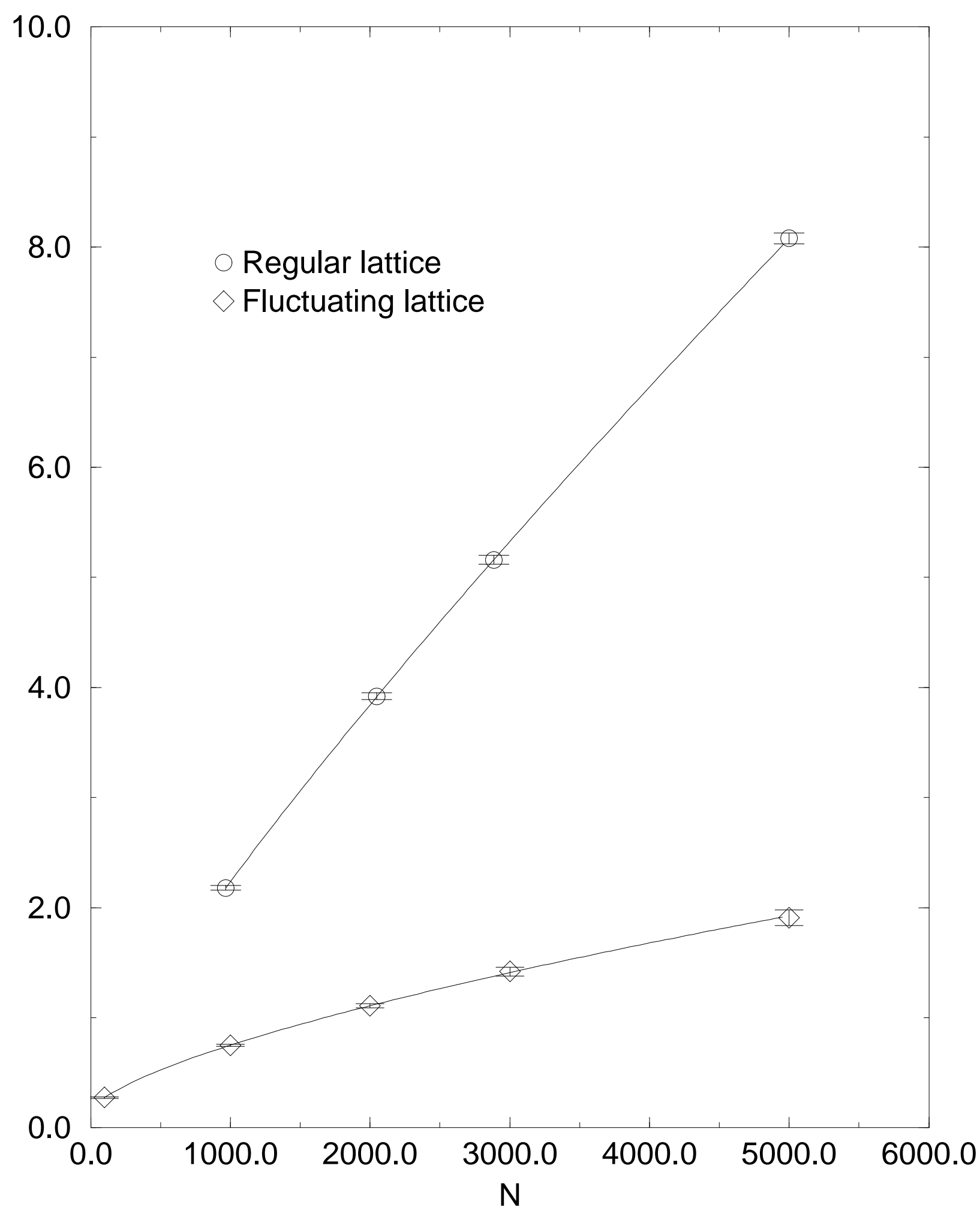


Regular lattice fits

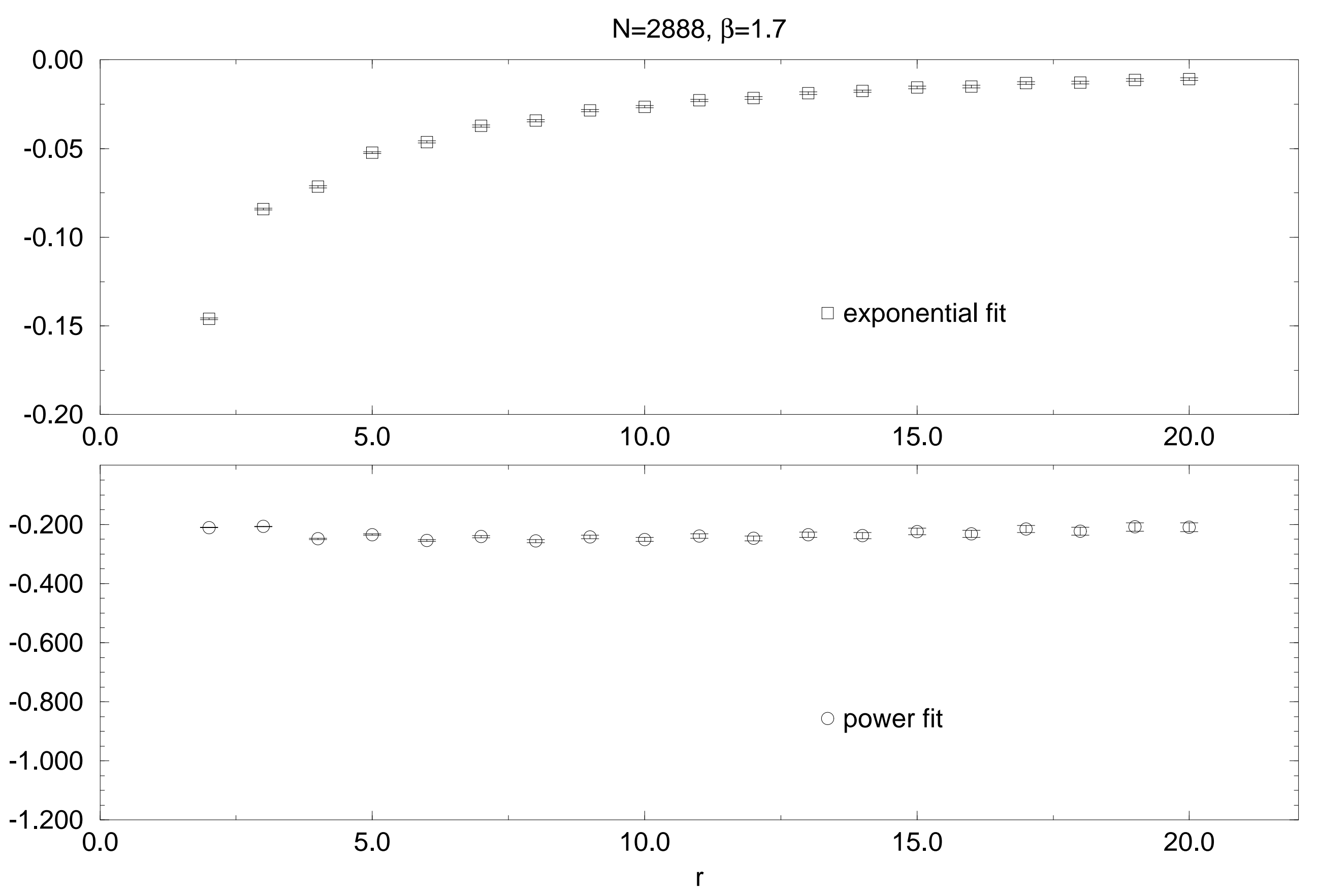




\section{Spin Correlators}
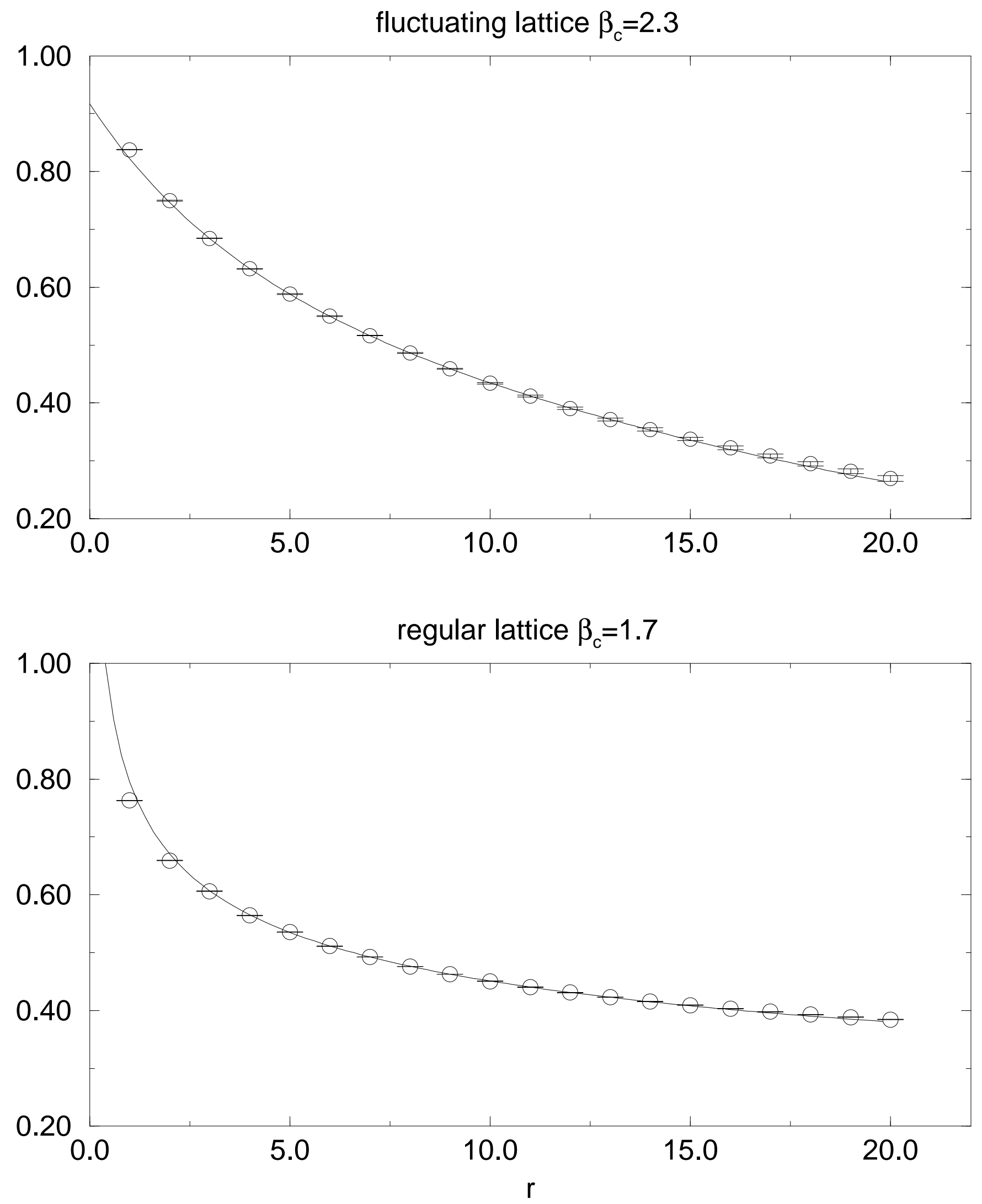
Fluctuating lattice fits

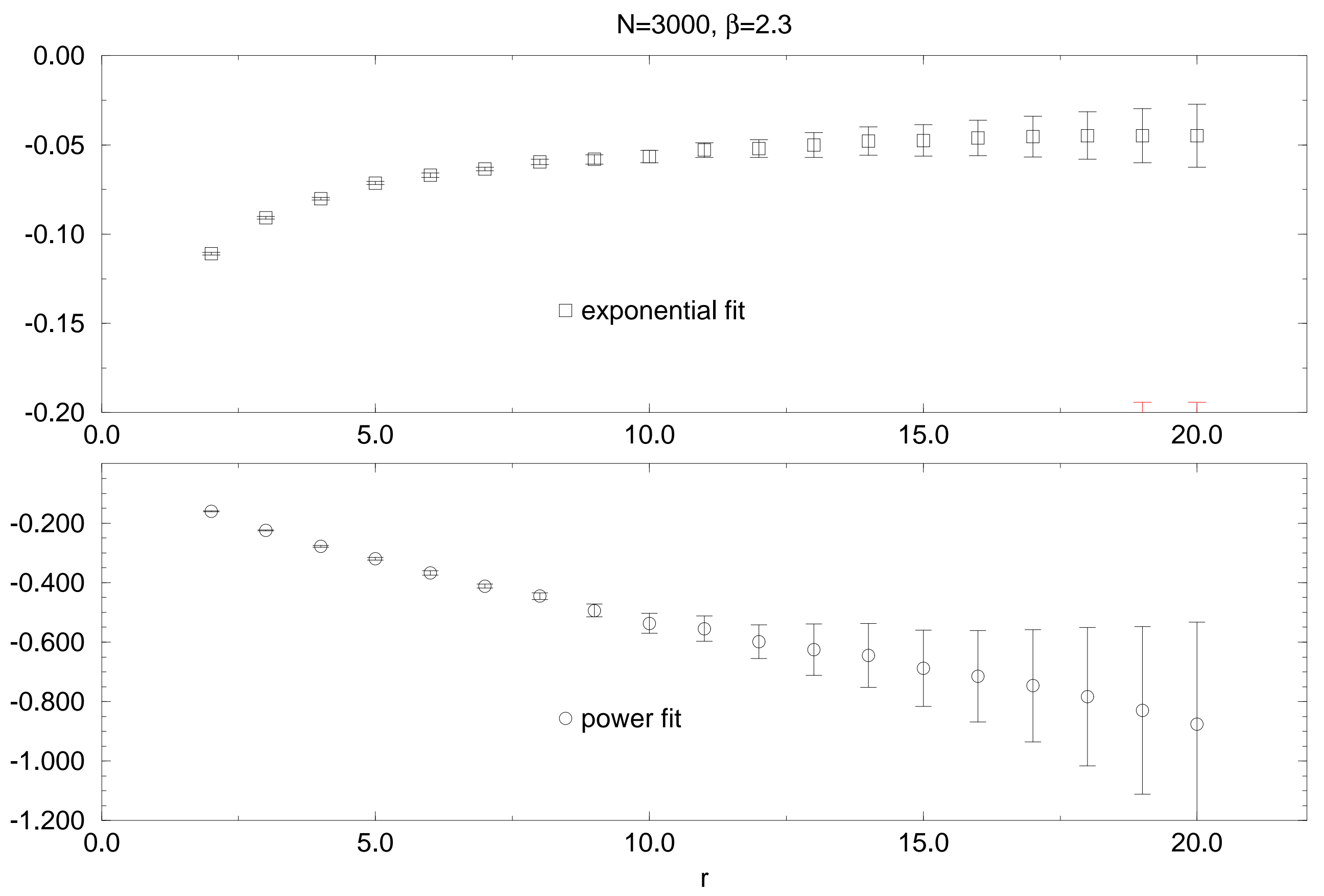




\section{Logarithm Spin Susceptibility}

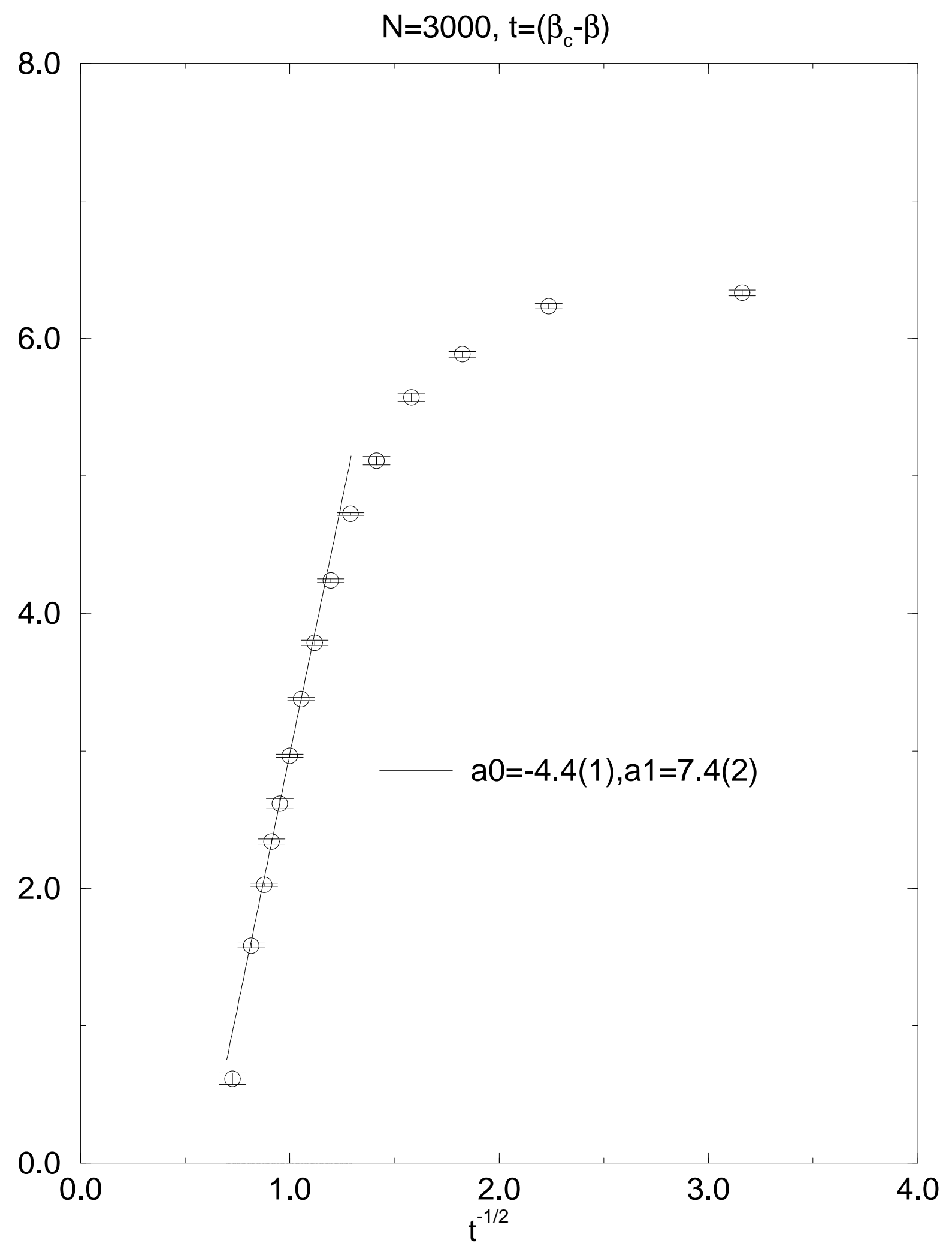



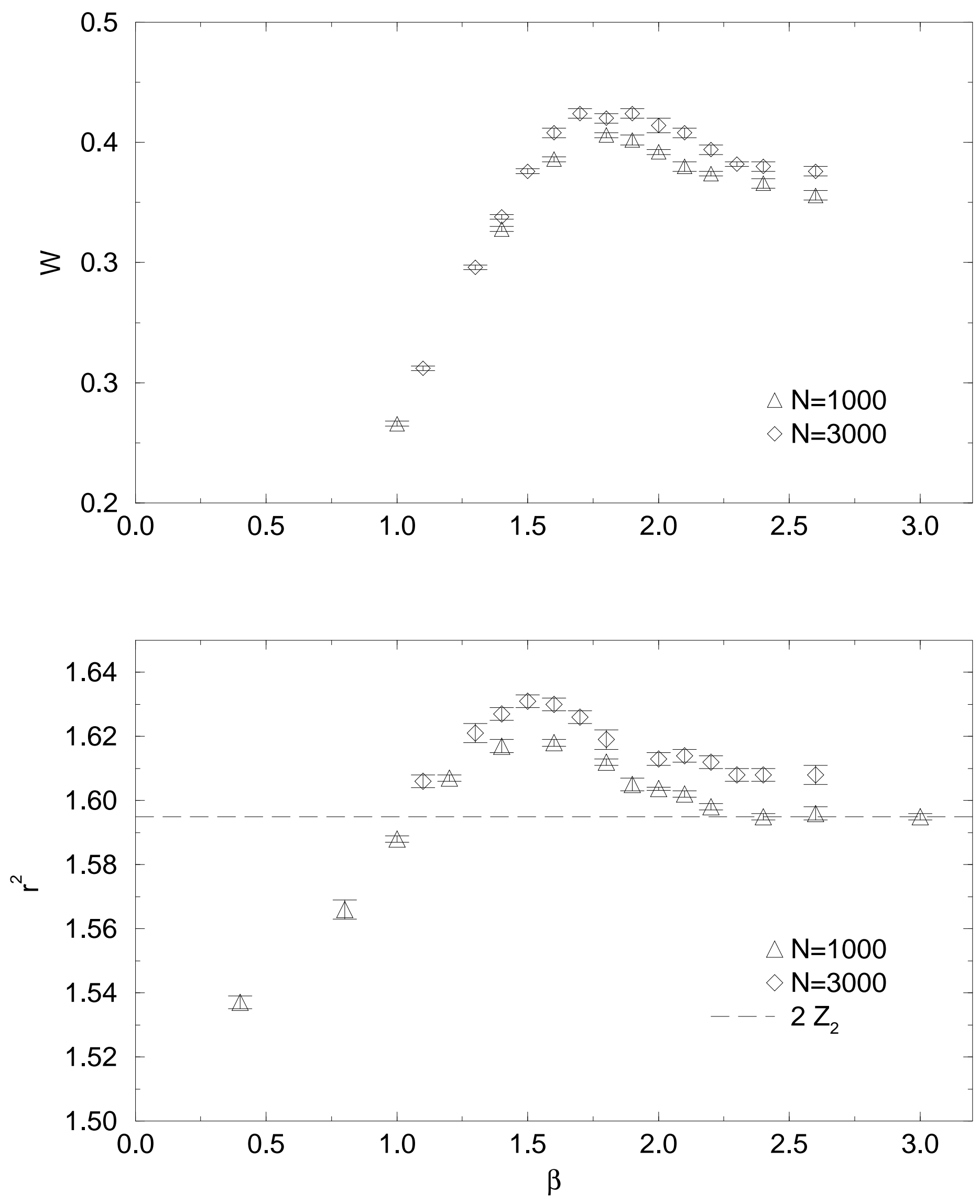

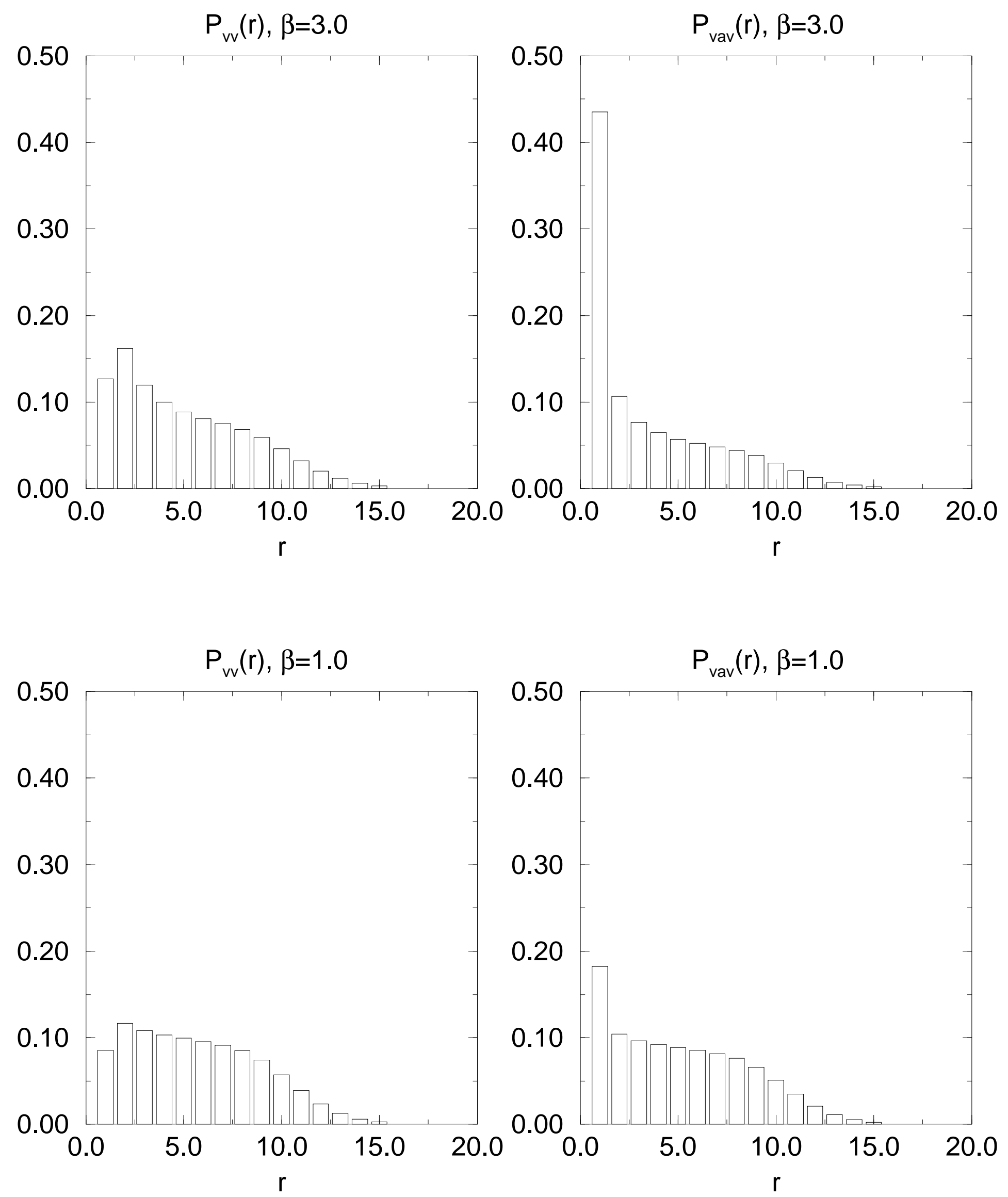